\title{
«Études Littéraires Africaines», Madagascar, textes réunis par Dominique Ranaivoson, n. 23
}

\section{Maria Clara Pellegrini}

\section{(2) OpenEdition}

1 Journals

\section{Edizione digitale}

URL: http://journals.openedition.org/studifrancesi/9053

DOI: 10.4000/studifrancesi.9053

ISSN: 2421-5856

\section{Editore}

Rosenberg \& Sellier

\section{Edizione cartacea}

Data di pubblicazione: 1 octobre 2008

Paginazione: 497-498

ISSN: 0039-2944

\section{Notizia bibliografica digitale}

Maria Clara Pellegrini, ««Études Littéraires Africaines», Madagascar, textes réunis par Dominique

Ranaivoson, n. 23», Studi Francesi [Online], 155 (LII | II) | 2008, online dal 30 novembre 2015, consultato il 13 janvier 2021. URL: http://journals.openedition.org/studifrancesi/9053 ; DOI: https://doi.org/ 10.4000/studifrancesi.9053

Questo documento è stato generato automaticamente il 13 janvier 2021.

\section{(c) (1)}

Studi Francesi è distribuita con Licenza Creative Commons Attribuzione - Non commerciale - Non opere derivate 4.0 Internazionale. 


\title{
«Études Littéraires Africaines», Madagascar, textes réunis par Dominique Ranaivoson, n. 23
}

\author{
Maria Clara Pellegrini
}

\section{NOTIZIA}

«Études Littéraires Africaines», Madagascar, textes réunis par Dominique RANAIVoson, n. 23, août 2007, pp. 110.

1 Rivista dell'APELA, Association pour l'Étude des Littératures Africaines fondata a Bordeaux nel 1984, «Études Littéraires Africaines» nasce come organo di informazione della produzione critica e letteraria africana, perseguendo l'obiettivo di una documentazione quanto più ampia delle diverse esperienze artistiche e scientifiche del continente, al di là delle divisioni linguistiche e culturali dei popoli che lo abitano o che, in qualche misura, ad esso appartengono, come le Antille e i paesi dell'Oceano Indiano.

Confrontandosi con la realtà plurale dell'Africa, ove convivono lingue, culture e storie diverse, la rivista si propone di restituirne la ricchezza, di rivelarne l'eterogeneità attraverso la scelta di un percorso monografico con il quale di volta in volta si indagano e si analizzano le produzioni di aree specifiche del continente, a partire dai contributi di specialisti che riferiscono della realtà africana, ora in veste di saggio ora di recensione breve. Al fine di offrire un inventario sempre aggiornato delle nuove proposte letterarie e scientifiche dei paesi africani, la rivista è divisa in due sezioni: una parte monografica seguita da una parte generale, «comptes rendus», nella quale sono esaminati testi editi nell'ultimo anno dell'Africa Nera francofona, anglofona, dell'Africa del Nord, delle Americhe e dell'Oceano Indiano.

Il numero 23 della rivista dedicato al Madagascar è stato realizzato con il contributo del critico malgascio Dominique Ranaivoson, specialista della letteratura malgascia di espressione francese. Dare visibilità a una produzione letteraria "minore" per 
diffusione, quale può essere considerata la letteratura in lingua malgascia, è l'obiettivo del dossier che analizza diverse realtà dell'isola: il teatro, la poesia, la prosa, i manuali scolastici, le riviste scientifiche universitarie e il fumetto alla luce della situazione politica ed economica ancora irrisolta della giovane repubblica, dei contrasti sociali ed etnici profondi e delle contraddizioni culturali di un paese diviso tra due lingue, il malgascio, lingua nazionale riscattata dal servaggio coloniale negli anni Settanta, e il francese traccia viva della colonizzazione. L'urgenza di uno studio che si interroga sulla situazione attuale della produzione in lingua malgascia prende rilievo di fronte alla scelta massiccia di scrittori e di intellettuali dell'isola di ricorrere alle edizioni francesi e inglesi per poter emergere oltre i confini di un'isola ancora sospesa nella celebrazione di un passato mitizzato, la cui memoria per le nuove generazioni è già logora, corrotta dalle colpe di un presente incapace di confrontarsi con la propria storia e con le rivoluzioni fallite messe sotto silenzio (1947). La lingua e la storia diventano le chiavi di lettura privilegiate per l'analisi della produzione dell'«isola continente», coma la definisce Dominique Ranaivoson, le linee guida di uno studio sulla letteratura locale tormentata dai fantasmi della questione identitaria, dal rifiuto e dell'accettazione della lingua del colonizzatore, minacciata dalle scarse risorse economiche che ne ostacolano la diffusione.

4 Il confronto con la produzione malgascia in lingua francese è inevitabile e doloroso nella misura in cui gli scrittori e gli intellettuali malgasci devono prendere atto del perdurare di una condizione di diffuso analfabetismo e di indigenza, di una povertà di risorse responsabili del ristagno dell'industria editoriale (Le paysage éditorial malgache, Claude RABENORO) dai quali sembrano immuni i soli generi della tradizione orale come il kabary (discorso pubblico) o lo hira gasy (sorta di operetta con canti e balli) sentiti e partecipati dalla maggioranza della popolazione. I due articoli sulla produzione letteraria in lingua malgascia che aprono e chiudono il dossier (La langue malgasy et les politique linguistiques di Dominique RANAIVOSON, Écrire en deux langues. L'expérience de JeanJoseph Rabearivelo et d'Esther Nirina di Claire RIFFARD) mettono in luce la fragilità del fenomeno letterario minacciato dal potere della lingua e della cultura francese alle cui lusinghe hanno ceduto poeti del passato come Rabearivelo e del presente come Raharimanana ed Esther Nirina nel tentativo di operare la conciliazione tra le due lingue.

5 Vengono altresì denunciate le difficoltà della produzione teatrale nel trovare spazi di diffusione e occasioni di rinnovamento tra gli scrittori di ultima generazione laddove la ristrettezza dei mezzi, sale piccole, mancanza di apparecchiature e di fondi, chiusura di teatri storici, sembra ostacolarne la fruizione, lasciando all'iniziativa di poche compagnie la possibilità di mantenere vivo tra la popolazione l'interesse per il genere (Le théâtre malgache contemporain: entretien avec Mbato Ravaloson).

6 Nell'ostacolare la diffusione dei testi in lingua malgascia, la povertà delle risorse pregiudica in modo ancora più rilevante lo sviluppo di una letteratura dalla vocazione politica e sperimentale la cui difesa rimane il baluardo di scrittori e di circoli che incoraggiano l'iniziativa degli autori emergenti (Sandrata, un groupe d'écrivains militants, José solofo). A dispetto di una produzione artistica e scientifica costretta all'esilio (Le paysage éditorial malgache, Claude RABENORO), sopravvive, invece, la letteratura in lingua malgascia di cultura popolare orale, come gli hain-teny, o il fumetto il cui successo si deve all'uso di un linguaggio che, nel rispetto del sentire tradizionale, è intriso di 
insegnamenti morali. Il dossier termina con una bibliografia di testi malgasci editi tra il 2006 e il 2007. 